\title{
Pathways to Biomedical Discovery*
}

\author{
Paul Thagard $\$$
}

A biochemical pathway is a sequence of chemical reactions in a biological organism. Such pathways specify mechanisms that explain how cells carry out their major functions by means of molecules and reactions that produce regular changes. Many diseases can be explained by defects in pathways, and new treatments often involve finding drugs that correct those defects. This paper presents explanation schemas and treatment strategies that characterize how thinking about pathways contributes to biomedical discovery. It discusses the significance of pathways for understanding the nature of diseases, explanations, and theories.

1. Introduction. Articles and textbooks in biochemistry, molecular cell biology, and biomedicine make frequent use of the concept of a biochemical pathway. This paper aims to answer fundamental questions about how knowledge of pathways contributes to biomedical discoveries concerning the causes and treatment of numerous diseases. These questions include: What are biochemical pathways? How are they represented so that scientists can reason about them? How do pathways furnish biological explanations of normal cellular function? How do pathways provide new mechanistic explanations of diseases? How does knowledge about pathways suggest novel treatments of diseases?

This paper concerns both the nature of biochemical pathways and the cognitive processes of scientists who use knowledge about them to make biomedical discoveries. I present a set of explanation schemas that characterize the typical patterns in which pathways explain how cells function and how diseases arise. Corresponding to the explanation schemas for

*Received July 2002; revised November 2002.

$\dagger$ To contact the author please write to Paul Thagard, Philosophy Department, University of Waterloo, Waterloo, ON, Canada N2L 3G1; e-mail: pthagard@uwaterloo.ca; web: http://cogsci.uwaterloo.ca.

$\$ I$ am grateful to Lindley Darden and two anonymous referees for very helpful suggestions. This research is supported by the Natural Sciences and Engineering Research Council of Canada.

Philosophy of Science, 70 (April 2003) pp. 235-254. 0031-8248/2003/7002-0001\$10.00

Copyright 2003 by the Philosophy of Science Association. All rights reserved. 
diseases are treatment strategies that suggest ways of curing or ameliorating the diseases by altering pathways. These treatment strategies provide medical researchers with cognitive pathways to new means of curing diseases.

2. What Are Pathways? According to the Oxford English Dictionary (second edition), the word "pathway" originated in the sixteenth century, but it only became biologically important in the 1920s. Around that time, the concept of a neural pathway was formed, meaning "a chain of nerve cells forming a continuous route along which impulses of a particular kind habitually travel." Also formed then was the concept of a biochemical pathway, "the sequence of reactions undergone by a compound or class of compounds in a natural environment, esp. a living organism." A reaction is "a chemical change, where the transformation of one or more components into new substances occurs, accompanied by energy changes" (Mandel 1969, 285).

Particularly important are metabolic pathways, which are sequences of chemical reactions that occur within living cells (Moran et al. 1994, ch. 14). In metabolism, the main relevant components are large molecules such as proteins, polysaccharides, and lipids, and the transformations involve their construction or decomposition. Some metabolic pathways are linear, proceeding in a step by step fashion from one molecule to another. But other pathways are cyclic, looping back to produce the chemicals that initiate them. Pathways can also have feedback loops, for example when the product of a pathway controls the rate of its own synthesis through inhibition of an early step (Moran et al. 1994, 14-11). Each pathway is organized by the links in its chemical reactions, with the product of one reaction providing a reactant that starts or an enzyme that catalyzes a subsequent reaction (Karp 2001).

Pathways are crucial to explaining how cells carry out their major functions, including energy acquisition, division, motion, tissue formation, signaling, and apoptosis (cell death). To stay alive and carry out their other functions, cells must constantly replenish their supply of energy furnished by ATP (adenosine triphosphate). In most cells, glucose $\left(\mathrm{C}_{6} \mathrm{H}_{12} \mathrm{O}_{6}\right)$ is a major source of energy, so the pathway that converts glucose, called glycolysis, is ubiquitous. This pathway converts one molecule of glucose into two molecules of the 3-carbon compound pyruvate, producing as well two molecules of ATP. The glycolytic pathway consists of ten chemical reactions catalyzed by enzymes. Many other pathways are involved in a cell's acquisition of energy and its performance of other functions. The next section will describe how pathways are used in mechanistic explanations of how cells carry out these functions.

For scientists to reason about pathways, they need representations of 
them, including both external representations via print and computer screens and internal mental representations. In order to describe the structure and dynamics of pathways, both verbal and visual representations are useful. Textbooks use a combination of verbal representations, for example the statement that glycolysis consists of ten chemical reactions, and visual representations. Moran et al. (1994, 15-4f.) provide long verbal descriptions of glycolysis, as well as a detailed two-page diagram of the molecules and steps involved in the transformation of glucose into pyruvate. Books only allow two-dimensional visual representations of molecules and their interactions, but it is becoming common for software packages and websites to have three-dimensional representations of complex molecules (e.g., Sapp 2002).

Karp (2001) describes EcoCyc, a superbly organized database that represents a total of 165 metabolic pathways for the bacterium E. coli. It contains a useful and easily browsed combination of verbal and twodimensional visual information about pathways, chemical reactions, and molecules. The database is structured as a set of frames, which are data structures widely used in artificial intelligence to represent concepts. Each frame represents a distinct biological object such as a gene or protein, with labeled connections that display the relations of the object such as its role in chemical reactions. Karp suggests that the database is an encoding of the biological theory of $E$. coli metabolism that is more comprehensive and easily used than any other available representation in computers or human minds.

I am unaware of any psychological studies of how people represent chemical reactions and pathways. I conjecture that some biochemists have mental representations of molecules that depict their three-dimensional structure. To capture the dynamics of pathways, it would be desirable to have animations that display a sequence of reactions, but I do not know whether humans or computers are currently capable of 2-D or 3-D animations of pathways. In any case, verbal and 2-D pictorial representations of pathways suffice for biological explanations.

3. How Pathways Explain. Articles and textbooks in molecular cell biology rarely if ever mention laws of nature, so the explanations they offer cannot be understood in terms of the deductive-nomological model of explanation that often applies in physics. But biochemical explanations frequently make reference to mechanisms, in keeping with the mechanism-based view of explanation espoused by such philosophers of science as Salmon (1984), Bechtel and Richardson (1993), and Machamer, Darden, and Craver (2000). I will now describe how biochemical pathways are a kind of mechanism, and how they provide mechanistic explanations of biological functions. 
Machamer, Darden, and Craver $(2000,3)$ characterize mechanisms as "entities and activities organized such that they are productive of regular changes from start or set-up to finish or termination conditions." In biochemical pathways, the entities are molecules and the activities are the chemical reactions that transform molecules into other molecules. In the glycolytic pathway, for example, the entities are molecules such as the initial glucose and the terminal pyruvate, as well as the numerous molecules produced and transformed during the ten chemical reactions in the pathway. Molecules and chemical reactions in cells are organized such that they are productive of regular changes, as in the repeated transformation of glucose to pyruvate. Moreover, for each pathway, we can specify the set-up conditions as the molecules that initiate the first reaction in the pathway, and the terminating conditions as the molecules that are produced by the last reaction in the pathway.

Figure 1 depicts a short pathway responsible for the formation of the neurotransmitter dopamine. Dopamine is produced from the amino acid tyrosine by two chemical reactions. An enzyme converts tyrosine into Ldihydroxyphenylalanine (L-dopa), which is then converted by another enzyme into dopamine. The arrows in figure 1 represent the chemical activity of the molecules that together with the enzymes produce new molecules. Thus biochemical pathways are mechanisms. As indicated earlier, however, pathways need not have the simple linear form A - > B - > C, because they can contain cycles and feedback loops. Thagard 2002b argues that mechanisms such as the dopamine pathway are a crucial part of mental computation.

Machamer, Darden, and Craver $(2000,22)$ argue that explanation is based, not just on regularity, but on revealing the productive relation in a mechanism. What explains is not regularities, but the activities that sustain regularities. Thus biochemical pathways explain by showing how changes within a cell take place as the result of the chemical activities of the molecules that constitute the cell. Such explanations are mechanistic because they are analogous to ones that apply to human-built machines with interrelated parts that combine to produce functional behaviors (Bechtel and Richardson 1993, 17).

The primary explanations in biochemistry answer how-questions rather

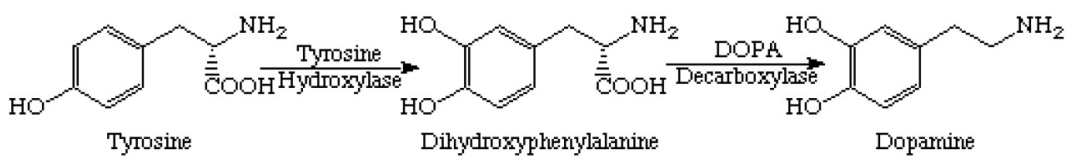

Figure 1. Diagrammatic representation of the pathway that produces dopamine. From Messer (2002). Reproduced by permission. 
than why-questions: How do cells get energy? How do cells divide? How do cells form tissues? Discovery of a pathway provides a mechanism that describes the productive activity that enables the cell to perform tasks that contribute to its own survival and to the survival of the organism of which the cell is a part. Table 1 reviews the major functions of cells along with the relevant how-questions and sample pathways that partially answer the questions.

For each of the functions in Table 1, we can ask the why-question: Why does a cell have that function? In some cases the answer is obvious: cells have the function of energy acquisition in order to carry out the chemical reactions necessary to sustain themselves, and they have the function of cell division in order to produce new cells and new organisms. Natural selection would quickly eliminate any organism that did not contain cells that effectively replenish energy and produce new cells. Even single-celled organisms such as bacteria have mechanisms for moving and sending chemical signals to each other, thereby improving their ability to find energy sources and avoid difficult environments. In multi-cellular organisms, natural selection has favored individuals with mechanisms for effectively joining cells into tissues and organs. It is more surprising that cells contain apoptotic pathways that lead to their own demise, but programmed cell death benefits an organism by eliminating defective or unnecessary cells. Questions about why cells contain particular biochemical pathways can thus be given functional explanations in terms of the contributions that the pathways make to cells' ability to carry out functions that contribute to the survival of organisms (on functions and functional explanations, see McLaughlin 2001 and Allen, Bekoff, and Lauder 1998).

TABLE 1. Cell Functions, How-Questions, and Examples of Biochemical Pathways That Help to Explain How the Cell Accomplishes the Function.

\begin{tabular}{|c|c|c|}
\hline Cell function & How-question & Sample pathway \\
\hline $\begin{array}{l}\text { Energy } \\
\text { acquisition }\end{array}$ & How do cells get energy? & Glycolysis \\
\hline $\begin{array}{l}\text { Mitosis } \\
\quad \text { (division) }\end{array}$ & How do cells divide? & APC pathway \\
\hline Motion & How do cells move? & $\begin{array}{l}\text { Ras-linked signal transduction } \\
\text { pathways }\end{array}$ \\
\hline Adhesion & How do cells adhere to form tissues? & Integrin signaling pathway \\
\hline Signaling & How do cells signal each other? & $\begin{array}{l}\text { Growth hormone signaling } \\
\text { pathway }\end{array}$ \\
\hline $\begin{array}{l}\text { Apoptosis } \\
\text { (cell death) }\end{array}$ & $\begin{array}{l}\text { How do cells provoke their own } \\
\text { destruction? }\end{array}$ & AKT signaling pathway \\
\hline
\end{tabular}

Many other pathways are described in Moran et al. 1994 and Lodish et al. 2000. For Web examples, see Biocarta 2002. 
In this paper, however, my concern is with mechanistic explanations using pathways rather than with functional explanations of why cells have pathways. Here is a general schema for such how-explanations, consisting of an explanation question and a pattern for answering that question:

\section{Cell Function Explanation Schema:}

Explanation question:

How does a cell accomplish a function that benefits the organism it is part of?

Explanation pattern:

The cell has available set-up molecules.

The cell can use terminating molecules to help accomplish the function.

A pathway links the set-up molecules to the terminating molecules by a sequence of chemical reactions.

Here the words in boldface are variables that need to be filled in for particular functions and pathways such as the ones mentioned in Table 1. The cell function explanation schema provides a general characterization of the structure of the mechanistic explanations that use pathways to explain how cells work.

This schema is an instance of the more general biological function explanation schema, which has the following structure:

Biological Function Explanation Schema

Explanation target:

Why does an organism have a mechanism?

Explanation pattern:

The mechanism enables the organism to accomplish some function that is important for its survival and reproduction.

So the organism has developed the mechanism as the result of natural selection.

Functional explanations are often stated in terms of entities, for example as answers to questions such as why animals have hearts. But entities such as hearts only have explanatory value as components of mechanisms to which they contribute activities, for example the heart's pumping blood. So I shall presume that functional explanations are directed toward the explanations of mechanisms that involve activities as well as the existence of entities (see Craver 2001 for a discussion of functions and mechanisms).

In the next section I will present explanation schemas that characterize how pathways can be used to explain why cells sometimes fail to work, leading to disease. Explanation schemas and similar abstractions have been discussed in philosophy and cognitive science using varying terminology (see, for example, Darden and Cain 1989, Giere 1999, Kitcher 1993, Leake 1992, Schaffner 1993, Schank 1986, and Thagard 1988, 1992). 
4. Pathways and the Explanation of Disease. According to the Oxford English Dictionary, a disease is a "condition of the body, or of some part or organ of the body, in which its functions are disturbed or deranged." Although there are still diseases such as Alzheimer's whose causes are unknown, medical science has identified the causes of many human diseases. The germ theory of disease developed by Pasteur in the 1860s has provided explanations of many infectious diseases such as tuberculosis. Understanding of Mendelian genetics made possible explanation of diseases caused by defective genes, beginning with alkaptonuria in 1902. In the 1910s and 1920s, nutritional diseases such as scurvy were identified as originating from vitamin deficiencies. During the 1950s, increased understanding of the immune system led to the realization that diseases such as lupus erythematosus are caused by attacks on the body by the immune system. In the early 1980s, cancer researchers discovered that the development of tumors is the result of successive damage to several genes. Some diseases have multiple causes, for example arteriosclerosis, which arises in patients with genetic tendencies such as inclination to hypertension and environmental factors such as high-fat diets. For a review of the explanation schemas used in these kinds of diseases, see Thagard 1999, ch. 2.

All of the discoveries of the basic causes of these kinds of diseases involved little detailed knowledge of the mechanisms of disease. Although the germ theory of disease explains why people get sick from bacterial and viral infections, it does not explain how micro-organisms invade the body and produce disease. Similarly, identifying scurvy as caused by vitamin C deficiency does not explain how lack of ascorbic acid leads to physical problems such as bone loss. But elucidation of the molecular mechanisms underlying disease has proceeded with increasing rapidity in the past few decades, by identifying key biochemical pathways that fill in the gap between the basic causes of infectious, genetic, nutritional, autoimmune, and cancer diseases and their symptoms.

Pathways explain how diseases arise by a general pattern that I shall term malfunctional explanation, in contrast to the functional explanations discussed in the last section. Malfunctional explanation also involves mechanisms. If my car fails to start, I explain the malfunction based on background knowledge of the entities and activities that normally lead from my turning the ignition key to the running of the engine. Failure is explained by some defect in the mechanism, including entities such as the battery and activities such as ignition. Malfunctional explanations fit the following general schema:

\section{Malfunctional Explanation Schema}

Explanation target:

Why does a system fail to function normally? 


\section{Explanation pattern:}

Normal function in the system is produced by a mechanism with a set of entities and activities.

The mechanism had defects in some entities and activities.

So the system cannot function normally.

This malfunctional explanation schema applies both to human artifacts such as cars and to biological organisms. For diseases, the schema can be instantiated by means of general causes such as infectious agents that cause defects in organs, but deeper understanding is achieved by bringing the schema down to the level of specific pathways in cells. Defects in entities usually lead to loss of activity, as when a car will not start because of a faulty battery, but they can sometimes result in excessive activity, as when a stuck gas pedal makes a car engine race. Woolfolk (1999) provides a useful survey of recent work on malfunctions and disease.

It is easy to find examples where understanding of pathways has contributed to mechanistic explanation of the origins of disease. For infectious diseases, we want to be able to explain both how the infectious agent invades the body and how it impedes bodily functions. Consider, for example, the bacterium Helicobacter pylori, which is now considered to be the cause of most peptic ulcers. This hypothesis was established in the 1980s and early 1990s on the basis of clinical trials that showed that antibiotic therapy that eliminated $H$. pylori usually cured ulcers (Thagard 1999). Only recently, however, has medical research arrived at an understanding of the pathways by which $H$. pylori inhabits gastric mucosa, induces apoptosis in gastric epithelial cells, and initiates inflammatory responses through immune system activity (Achtman and Suerbaum 2001). Similarly, understanding of viral infections such as HIV is now deepened by appreciation of the chemical reactions that enable viruses to bind to the surfaces of cells and to reproduce by exploiting internal cell pathways.

Other kinds of diseases have also increasingly received analysis at the molecular level. We now know, for example, how scurvy arises from deficiency of ascorbic acid, which is essential for a biochemical pathway that produces collagen, the protein that is the main constituent of connective tissue and bones. For many genetic diseases there is increasing understanding of how genetic defects cause malfunctions. For example, in Huntington's disease, a fatal neurodegenerative disorder, a mutated gene produces an abnormal version of a protein that contributes to apoptosis in brain cells. The mechanisms of autoimmune diseases are also becoming understood; some may arise from mutations in immune system cells that prevent them from dying after they have dealt with infectious agents, so that the immune cells attack cells from the body.

The medical importance of biochemical pathways is particularly clear in the understanding of different kinds of cancer. Hahn and Weinberg 
(2002a) summarize the limited number of mechanisms that they think govern the emergence of cancers from normal tissues, including: tumorsuppressor pathways such as RB and p53; telomere shortening pathways that cause apoptosis; signaling pathways such as ras. Perturbation of these pathways allows transformation of human cells that grow out of control and form cancerous tumors. Additional pathways are involved in the ability of some tumors to recruit blood supplies (angiogenesis) and spread through the body (metastasis). Hahn and Weinberg have made available on the Web a superb "subway map" of cancer pathways that illustrates the interacting genetic and chemical mechanisms involved in the development of cancer (Hahn and Weinberg 2002b; see Figure 2).

These examples illustrate different roles of biochemical pathways in explaining disease. As in all malfunctional explanations, explanations of disease involve a disruption of function, but pathways can contribute to disruption in different ways, involving both decrease and increase of ac-

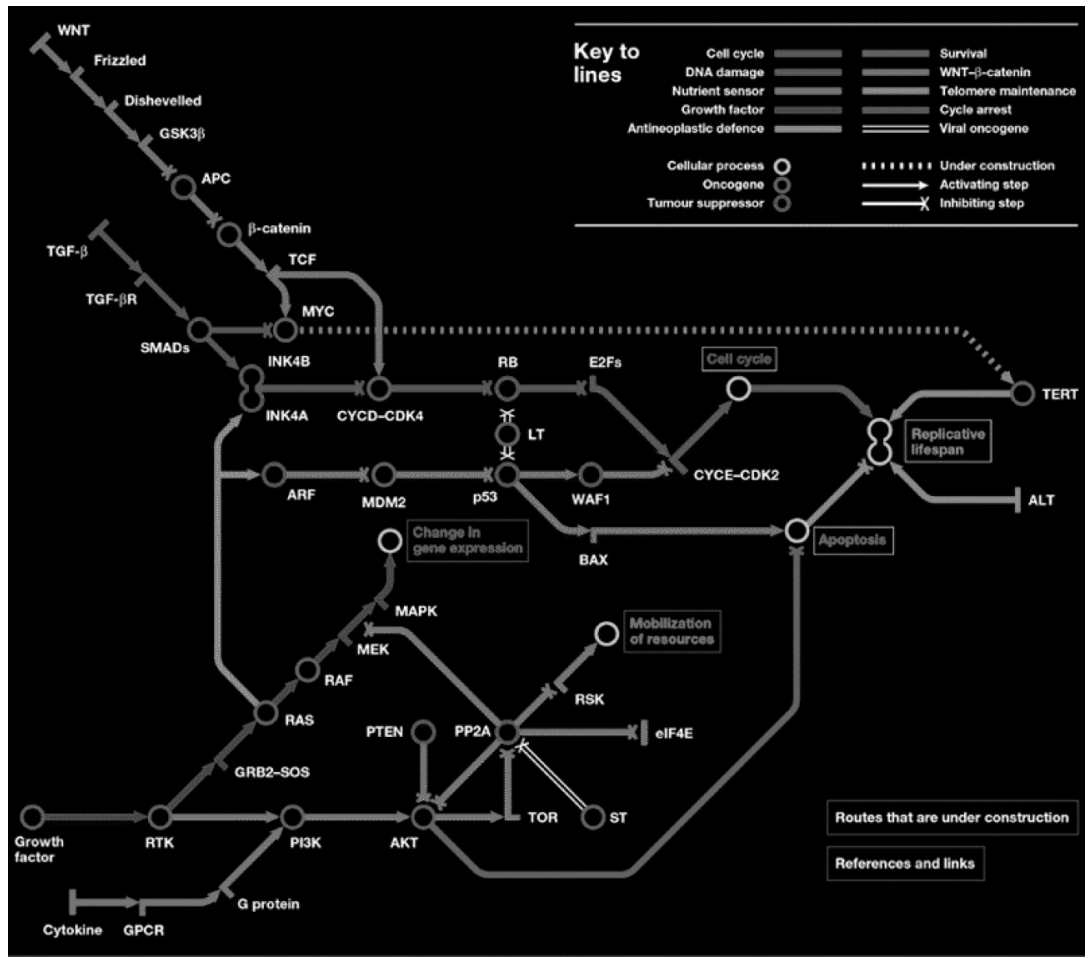

Figure 2. Map of cancer pathways, from Hahn and Weinberg (2002b). Reprinted by permission from Nature Reviews Cancer, copyright (2002) Macmillan Magazines Ltd. 
tivity. The most obvious is when a pathway is the mechanism that enables a cell to carry out some useful function, such as producing collagen or controlling cell proliferation. Defects in such pathways consist of defects in entities such as genes needed to produce proteins that regulate cell division. The general explanation schema is:

\section{Pathway Defect Explanation Schema \\ Explanation target:}

Why does a cell become defective in a function?

Explanation pattern:

The cell carries out the function via a pathway.

The pathway is defective in some molecules and reactions.

So the cell cannot carry out its function.

Instantiation of this schema requires identifying the relevant pathway.

Figure 1 displayed the pathway that produces dopamine from L-dopa. In patients with Parkinson's disease, neurons in the substantia nigra that normally produce dopamine fail to do so, leading to severe problems in motor control. Thus Parkinson's disease instantiates the pathway defect explanation schema because it is explained by a decrease in activity of the dopamine pathway (Chesselet and Delfs 1996). Dopamine defects can also cause disease derived from increase in pathway activity: an overactive dopamine system is implicated in schizophrenia (Carpenter and Buchanan 1994).

The pathway defect explanation schema fits well with explanations in cancer, genetic, and nutritional diseases that involve the disruption of functional pathways, but it does not capture how pathways contribute to explanation of infectious and autoimmune diseases. We can distinguish between two kinds of malfunctional explanations, internal and external. In the internal kind, the malfunction is the result of something going wrong inside an entity, for example when a car's battery wears out. The pathway defect explanation schema is internal in this way, since it involves pathways inside a cell that break down. In diseases caused by bacterial infection, however, the crucial pathways partly involve how the infectious cells wreak their damage on other cells. Similarly, the relevant pathways in autoimmune diseases include not only the internal ones of the cells that are damaged but also the genetically defective pathways of the immune cells that attack them.

We thus need a general schema that describes how pathways in cells external to functional cells can be used in the explanation of disease:

External Pathway Explanation Schema:

Explanation target:

Why does a cell become defective in a function? 


\section{Explanation pattern:}

The cell is destructively affected by external agents, such as bacteria, viruses, or autoimmune cells.

These external agents operate by means of pathways that enable them to invade and disrupt the cell.

So the cell becomes defective and cannot carry out its function.

Understanding these kinds of pathway-based explanations of disease is important for analyzing the cognitive strategies involved in discovering new treatments for disease.

5. Pathways and the Treatment of Disease. Current biomedical research abounds with investigations of pathways, partly in order to increase understanding of the mechanisms responsible for diseases, but primarily in order to find new treatments for them. This section shows how an understanding of pathways often leads to new medical treatments. The previous section described how mechanistic explanation of diseases can be based either on defective internal pathways or on destructive external pathways. Treatments for diseases can therefore consist either of repairing defective internal pathways or of blocking destructive external pathways. Repairing defective pathways can involve either enhancing them to restore an insufficiently active pathway, or inhibiting them to stop an overactive pathway. I will now describe cognitive strategies for finding pathway-based treatments for disease.

5.1. Enhancing Pathways. Many diseases are caused by defective internal pathways inside cells and are therefore potentially treated by changing the relevant molecules and reactions. The most obvious examples are diseases caused by single-gene defects such as cystic fibrosis and Huntington's disease. Cystic fibrosis, the most common inherited disease among Caucasians, involves the production of abnormal secretions in airway epithelia and elsewhere. In the 1980s, the discovery was made that cystic fibrosis is caused by mutations in the gene CFTR, which encodes a protein needed to maintain a balance of sodium and chloride in cells (Lodish et al. 2000, 597). Attempts are underway to find viral and other vectors that can transport normal CFTR genes into cells in order to restore normal function. The treatment strategy can be characterized as follows:

\section{Gene Therapy Treatment Strategy}

Treatment question:

How can a disease caused by a defective gene be treated?

Treatment discovery pattern:

Identify the defective gene and the defective cellular pathway that it affects.

Determine how to insert normal genes into cells to fix the pathway. 
To date, gene therapy has had little success in curing diseases such as cystic fibrosis, in part because of difficulties using viral vectors to insert genes into cells. The applicability of gene therapy may be limited by the diversity of phenotypic expression and the operation of other genetic mechanisms such as imprinting in diseases like cystic fibrosis and Huntington's. But hundreds of clinical trials using gene therapy on many diseases are now under way.

Defective genes are an extreme case where treatment is needed to enhance pathway function. Some diseases arise when pathways are not functioning to the extent that they should, and drug treatments can work to enhance pathway activity. An example is the recently approved class of antidiabetes drugs, thiazolidinediones (brand names Avandia and Actos). These drugs reduce cells' acquired resistance to insulin by activating $\operatorname{PPAR} \gamma$, a peroxisome proliferator receptor. The peroxisome in a cell is responsible for many functions such as the breakdown of fatty acids. Stimulation of the PPAR $\gamma$ pathway improves the ability of cells to use insulin to incorporate glucose from the blood stream. Here is the general strategy:

\section{Pathway Stimulation Treatment Strategy}

Treatment question:

How can a disease affected by an underactive pathway be treated?

Treatment discovery strategy:

Determine the molecules and reactions in the pathway.

Identify a molecule in the pathway susceptible to increased activity.

Search for drugs that increase the activity of the molecule.

This strategy is currently being used to look for cancer treatments that restore the function of defective pathways for apoptosis and tumor suppression, for example the p53 pathway (Hupp, Lane, and Ball 2000).

5.2. Inhibiting Pathways. The most common strategy for treating disease is not to enhance pathways, but rather to block pathways whose activity produces the disease. Gene therapy is not the only way of dealing with the pathways of genetic diseases, because it may also be possible to intervene at later stages in the pathways. For example, there is recent excitement concerning the prospect of treating Huntington's disease, not by replacing the mutant gene that causes it, but by counteracting the effect of the defective protein that the gene produces (Steffan et al. 2001). Other pathway alterations have already been used to treat important diseases. Patients with Parkinson's disease are given L-dopa to enable the body to produce dopamine needed by motor neurons, and medical researchers continue to search for new ways to stimulate dopamine pathways.

Statins are drugs that are very widely prescribed for reduction of high 
cholesterol levels, which are implicated in arteriosclerosis and heart disease. Most cholesterol in the body is produced by the liver, through a pathway that includes a feedback loop: cholesterol inhibits an enzyme, HMG Co-A reductase, that controls one of the crucial chemical reactions in the cholesterol pathway. In the 1960s, researchers attempted to find a substance that would inhibit the action of this enzyme, examining more than 6,000 microbes (Endo 1992). Two antifungal substances produced by molds were found to have the desired property, and other, more effective statins have been developed based on these substances. They are very useful for people whose cholesterol pathway is defective in the sense that it produces unhealthy levels of cholesterol. The treatment strategy here is as follows:

\section{Pathway Inhibition Treatment Strategy}

Treatment question:

How can a disease be treated by inhibiting a pathway?

Treatment discovery strategy:

Identify a pathway whose activity contributes to the disease.

Determine the molecules and reactions in the pathway.

Search for drugs that inhibit the pathway by suppressing molecules and reactions.

Suppression of molecules and reactions can involve either using a drug to reduce available amounts of a molecule that serves as a reactant or enzyme, or using a drug to block a receptor molecule required for a reaction to occur. Treatments for schizophrenia involve drugs that block the operation of dopamine by binding to dopamine receptors on neurons and thereby preventing the operation of dopamine-triggered pathways within the neurons.

The discovery of the breakthrough cancer drug Gleevec is another instance of this strategy (NCI 2002). Gleevec has provided a remarkably effective treatment for a serious blood cancer, chronic myelogenous leukemia. In the 1980s, researchers found that the cancerous cells in this disorder contain an abnormal bcr-abl gene that is supposed to encode an enzyme, specifically a tyrosine kinase, that helps to regulate cell growth and division. The aberrant bcr-abl gene floods the cell with the instruction to divide constantly. Independently, scientists were looking for agents to inhibit protein kinases, and had found a chemical called STI571. This chemical was shown to inhibit the protein produced by the bcr-abl gene and halt the growth of leukemia cells. After clinical trials showed the chemical to be highly effective in people, it was renamed Gleevec and is now commercially available. Just as statins intervene in a pathway to reduce cholesterol production, Gleevec intervenes to reduce chemical signaling that makes cells grow out of control. 
Similarly, the drug Herceptin, used for metastatic breast cancer, is a monoclonal antibody that aims at $\mathrm{Her} 2 / \mathrm{Neu}$, a tyrosine kinase receptor. Herceptin restricts growth of breast cancer cells by blocking this receptor and thereby limiting signals that stimulate cell growth. Yet another example of pathway inhibition is the treatment of depression by serotonin reuptake inhibitors such as Prozac. They slow the uptake of serotonin by nerve cells, making more of the neurotransmitter available; and they also have an internal enhancing effect on neurons by increasing the availability of brain derived neurotrophic factor (BDNF).

Sometimes investigation of pathways can provide an understanding of why an old drug works. Salicylic acid has been used for centuries to reduce pain, but its mechanism of operation was only identified in 1971, when John Vane discovered that aspirin inhibits prostaglandins. This discovery also explained why aspirin sometimes causes stomach problems, because prostaglandins also serve to protect the stomach lining. Subsequent research concerning the pathways that produce prostaglandins identified two enzymes, COX-1 and COX-2, with only the latter triggering inflammation. In the 1990s drugs were found that inhibit only COX-2 activity, and these are now widely used for treatment of arthritis.

A similar discovery route involves two classes of drugs for the treatment of high blood pressure, ACE inhibitors and angiotensin receptor antagonists. These drugs intervene at different steps in the renin angiotensin enzymatic pathway, which produces angiotensin II, a chemical important for blood pressure control. ACE inhibitors have been widely used, but in some patients they produce a cough as the result of reducing the breakdown of bradykinin. The newer angiotensin receptor antagonists intervene in the crucial pathway at a later stage, blocking the binding of angiotensin II to AT1 receptors that cause constriction of blood vessels. These two treatments illustrate two different ways in which a pathway can be inhibited: ACE inhibitors suppress an enzyme, while angiotensin receptor antagonists block a receptor (Therapeutics Initiative 2002).

One of the most successful drug treatments in recent years has been the use of protease inhibitors to prevent or retard the development of AIDS caused by HIV. This virus has nine genes including one that codes for HIV protease, an enzyme that is crucial for the formation of new viral particles within infected cells. Drugs have been found that inhibit the operation of HIV protease so that the virus does not reproduce. Research is also underway to find drugs that interrupt the mechanism by which viruses dock with cells, preventing them from becoming infected at all. Similarly, the search is underway for pathways in infectious bacteria that might be disrupted in order to kill them without bacteria developing resistance. 
5.3. Exploiting External Pathways. Pathway inhibition and enhancement are the most common ways to treat diseases, but a third strategy is available for cancer cells that have defective pathways. Instead of trying to fix the pathways, therapies are being devised to exploit the fact that the cancer cells are different from ordinary cells and therefore susceptible to special destructive agents. The hope is to find a treatment that destroys only cancer cells, unlike radiation and traditional chemotherapy which kill normal cells as well as cancer. One possibility currently under investigation is the use of oncolytic viruses that differentially infect cancer cells and kill them. For example, ONYX-15, a genetically engineered virus designed to infect cells with a defective p53 pathway, has shown some promise. In addition, a naturally occurring virus, the reovirus, has been found to infect and kill cancer cells with an activated ras pathway (Thagard 2002a). It remains to be seen how effective these treatments will prove in humans, but they add a novel strategy to the treatment of cancers by exploiting pathway activities peculiar to cancer cells. Here is a schema for this new kind of strategy:

\section{Pathway Exploitation Treatment Strategy \\ Treatment question:}

How can a disease be treated by exploiting a pathway?

Treatment discovery strategy:

Identify a pathway found in defective cells that contribute to the disease. Determine the molecules and reactions in the pathway.

Search for viruses that exploit the pathway and kill the defective cells.

Like all the other treatment discovery strategies and pathway explanation schemas I have discussed, this strategy requires identifying the regular pathway. Darden (2002) discusses general strategies for discovering mechanisms, including schema instantiation, modular subassembly, and forward and backward chaining; it would be interesting to explore whether these strategies are useful for discovering medically relevant pathways.

6. Diseases, Explanations, and Theories. This paper is primarily concerned with the local issue of how knowledge of biochemical pathways contributes to the explanation and treatment of disease, but it has implications for important global issues in the philosophy of science and medicine. I will now consider what reasoning about pathways tells us concerning the nature of diseases, explanations, and theories.

6.1. Disease. There has been considerable debate in the philosophy of medicine concerning the concept of disease (Caplan 1992; Caplan, Engelhardt, and McCartney, 1981; Humber and Almeder 1997; Reznek 1987). 
Boorse (1977) defends a naturalistic view of diseases as involving interference with normal functioning. In contrast, Englehardt (1984) advocates a normative account of diseases as involving judgments about human goals and the need for medical intervention. More radically, Hesslow (1993) argues that the concept of disease is more misleading than helpful in medical decision making. Resolution of the large issue of what diseases are is beyond the scope of this paper, but I will try to indicate how attention to biochemical pathways helps to clarify the issue.

As we saw in Section 4, pathway explanations operate at the level of cells rather than at the level of whole organs or complete organisms. At the level of organisms, the notion of function can indeed take on a normative dimension; for example, being farsighted is abnormal but is not a problem to someone who does not desire to read or do work that requires fine visual discrimination. But at the cellular level, normal functioning can be characterized in purely biological terms by answering the question: what are the biochemical pathways that universally operate in particular types of human cells to enable them to perform energy acquisition, mitosis, motion, adhesion, signaling, and apoptosis? Once these pathways are identified, abnormality can be recognized as a biological notion, just as Boorse suggests. Hence when the explanation and treatment of disease operates at the level of biochemical pathways, it provides support for the naturalistic, nonnormative conception of disease. However, not all medicine operates at the pathway level, and I leave open the possibility that a more general conception of disease may need to take into account valuations as well as biological explanations (Caplan 1992).

6.2. Explanation. I have described pathway explanations in terms of mechanisms, but have not addressed the general question of the compatibility of the mechanistic account of explanation with the traditional deductive-nomological model of explanation (Hempel 1965; see Salmon 1989 for a historical review). On the D-N model, an explanation answers a why-question by means of an argument in which the premises are a set of laws and initial conditions and the conclusion follows deductively from the premises. A proponent of this model might contend that it applies to pathway-based explanations because descriptions of chemical reactions that constitute pathways are laws of nature and the outcomes to be explained are derived deductively from them. Even if we do not usually have enough information to do a complete derivation, the pathway explanation could at least be seen as an explanation sketch that approximates a deductive explanation.

However, I do not find this attempt to fit mechanistic pathway explanations into the D-N model plausible. According to the ontic conception 
of explanation, to explain an event is to exhibit it as occupying its place in the discernible patterns of the world (Salmon 1984, 18); this is different from the epistemic conception, which holds that to explain an event is to show why it was to be expected. The chemical reactions in biochemical pathways are rarely stated as laws of the form: "Whenever there are such and such molecules together they are transformed into such and such molecules." Rather, as Section 2 argued, the point of describing pathways is not to state laws that yield deductive predictions but rather to specify a mechanistic pattern of entities and activities. Noting patterns of normal functioning, as well as deviations from them in the form of defective entities or activities, is what constitutes medical explanations.

In Section 3, I stated that the primary explanations in biochemistry answer how-questions rather than why-questions. It might be possible to construct a set of why-questions that correspond roughly to how-questions concerning cell functioning. For example, the question "How do cells get energy?" that is answered in part by the glycolytic pathway has the corresponding question "Why do cells have a glycolytic pathway?" But there does not appear to be a general reduction of how-questions to whyquestions, and the focus of the two kinds of question is different. Howquestions are more comprehensive than why-questions and are best answered by specifying one or more mechanisms understood as organized entities and activities. For medical explanations, a how-question is best answered not by citing a single pathway but by describing multiple interacting pathways. In Table 1, the sample pathways listed are only a few of the many pathways involved in energy acquisition, cell division, and the other cell functions. Thus answering a how-question is not a matter of assembling discrete arguments that can provide the answer to individual why-questions, but rather requires specification of a complex mechanism consisting of many parts and interconnections.

6.3. Theories. The deductive-nomological model of explanation is an elegant complement to the syntactic view of theories as sets of propositions in a formal language such as predicate calculus. But the mechanistic explanations found in biochemistry and biomedicine suggest that these fields need an account of theories different from the syntactic view. Theories of cell function and disease development are not naturally stated as sets of axioms, so what are they? The answer to this question is best approached through the cognitive view of theories, according to which a theory consists of interrelated mental representations and processes by means of which scientists solve problems (see Thagard 1988, 1992; Giere 1988, 1999). On this view, an explanation is a psychological process that fits something to be explained into a pattern established by the mental representations. In 
order to see the relation between this cognitive view of explanation and the ontic, mechanistic view advocated in the last section, we need an account of the relation between mechanisms and mental representations.

In Section 2, I described how textbooks present pathways using both verbal and visual representations. Verbal representations consist of both concepts such as glucose and rule-like propositions such as that glucose can be transformed by transfer of a phosphoryl group from ATP. In theory, pathways could be represented only verbally, but textbooks and articles abound with pictorial representations that are much better at conveying the organization of both entities and activities. The relevant entities are molecules whose chemical structure is crucial to their transformation into new molecules, whose spatial organization is also best represented visually. The activities are the sequences of chemical reactions that transform molecules, and the organization of these sequences is also well represented visually as seen in the dopamine pathway in Figure 1. Depiction of multiple interacting pathways, for example in the cancer map of Hahn and Weinberg (2002b), benefits especially from visual representations (Figure 2).

It is therefore plausible that the mental representations of pathway mechanisms that support cognitive processes include visual representations as well as verbal ones. Hence, on the cognitive view, a theory in biochemistry and biomedicine is a mental structure that describes mechanisms using visual as well as verbal representations. These structures enable minds to fit phenomena into the discernible patterns of the world as suggested by the ontic conception of explanation. This is how the cognitive view of theories meshes with the mechanism-based view of explanation.

7. Conclusion. Although successful drug treatments are still sometimes found by serendipity or massive screening of substances, drug discovery is increasingly performed by exploiting knowledge of the biochemical pathways that are responsible for the production of diseases. Pathwaybased strategies are becoming more and more feasible as information about the structure and function of genes, proteins, and reactions rapidly increases and becomes available in computerized databases. It should become possible to automate, at least in part, the use of pathways to identify potentially useful new treatments.

My goal in this paper has been to elucidate the nature of biochemical pathways and the mechanistic explanations they provide. I have described how pathway-based explanations of disease have frequently led to new treatments that diminish disease by enhancing or inhibiting pathways. In addition to characterizing the main pathway-based schemas for explaining diseases as mechanical malfunctions, I have identified several strategies for using knowledge of pathways to treat diseases. The results of this 
investigation are relevant to the philosophy of science in general, because they show how explanations can be based on mechanisms rather than covering laws, and they illustrate how mechanism-based explanations can naturally be translated into technological applications.

We have seen that a biochemical pathway is a sequence of chemical reactions that together accomplish a function within a cell. Similarly, a neural pathway is a sequence of neuron firings that accomplish a neurological function. Analogously, we can think of a cognitive pathway as a sequence of mental operations that accomplish an intellectual task. The explanation schemas and treatment strategies described in this paper provide initial characterizations of cognitive pathways to biomedical discovery.

\section{REFERENCES}

Achtman, Mark, and Sebastian Suerbaum (eds.) (2001), Helicobacter pylori: Molecular and Cellular Biology. Oxford: Horizon Scientific Press.

Allen, Colin, Marc Bekoff, and George Lauder (eds.) (1998), Nature's Purposes. Cambridge, MA: MIT Press.

Bechtel, William, and Robert C. Richardson (1993), Discovering Complexity. Princeton: Princeton University Press.

Biocarta (2002), "Charting Pathways of Life", http://www.biocarta.com/genes/index.asp.

Boorse, Christopher (1977), "Health as a Theoretical Concept", Philosophy of Science 44: $542-573$.

Caplan, Arthur L. (1992), "If Gene Therapy Is the Cure, What Is the Disease?", in George J. Annas and Sherman Elias (eds.), Gene Mapping: Using Law and Ethics as Guides. Oxford: Oxford University Press.

Caplan, Arthur L., H. Tristram Engelhardt, and James M. McCartney (eds.) (1981), Concepts of Health and Disease: Interdisciplinary Perspectives. Reading, MA: AddisonWesley.

Carpenter, William T., and Robert W. Buchanan (1994), "Schizophrenia", New England Journal of Medicine 330: 681-690.

Chesselet, Marie-Françoise, and Jill M. Delfs (1996), "Basal Ganglia and Movement Disorders: An Update", Trends in Neurosciences 19: 417-422.

Craver, Carl F. (2001), "Role Functions, Mechanisms, and Hierarchy", Philosophy of Science 68: 53-74.

Darden, Lindley (2002), "Strategies for Discovering Mechanisms: Schema Instantiation, Modular Subassembly, Forward/Backward Chaining", Philosophy of Science 69 (Proceedings): S354-S365.

Darden, Lindley, and Joseph Cain (1989), "Selection Type Theories", Philosophy of Science 56: $106-129$.

Endo, Akira (1992), "The Discovery and Development of Hmg-Coa Reductase Inhibitors", Journal of Lipid Research 33: 1569-1582.

Engelhardt, H. Tristram (1984), "Clinical Problems and the Concept of Disease", in Lennart Nordenfelt and B. Ingemar B. Lindahl.(eds,), Health, Disease, and Causal Explanations in Medicine. Dordrecht: D. Reidel.

Giere, Ronald N. (1988), Explaining Science: A Cognitive Approach. Chicago: University of Chicago Press. (1999), Science without Laws. Chicago: University of Chicago Press.

Hahn, William C., and Robert A. Weinberg (2002a), "Modelling the Molecular Circuitry of Cancer", Nature Reviews Cancer 2: 331-334.

(2002b), "A Subway Map of Cancer Pathways", http://www.nature.com/nrc/ journal/v2/n5/weinberg_poster/.

Hempel, Carl G. (1965), Aspects of Scientific Explanation. New York: The Free Press. 
Hesslow, Germund (1993), "Do We Need a Concept of Disease?", Theoretical Medicine 14: $1-14$.

Humber, James M., and Robert F. Almeder (eds.) (1997), What Is Disease? Totowa, NJ: Humana Press.

Hupp, Ted R., David P. Lane, and Kathryn L. Ball (2000), "Strategies for Manipulating the P53 Pathway in the Treatment of Human Cancer", Biochemical Journal 352: 1-17.

Karp, Peter (2001), "Pathway Databases: A Case Study in Computational Symbolic Theories", Science 293: 2040-2044.

Kitcher, Philip (1993), The Advancement of Science. Oxford: Oxford University Press.

Leake, David B. (1992), Evaluating Explanations: A Content Theory. Hillsdale, NJ: Erlbaum.

Lodish, Harvey, Arnold Berk, S. Lawrence Zipursky, Paul Matsudaira, David Baltimore, and James Darnell (2000), Molecular Cell Biology, 4th ed. New York: W. H. Freeman.

Machamer, Peter, Lindley Darden, and Carl F. Craver (2000), "Thinking About Mechanisms", Philosophy of Science 67: 1-25.

Mandel, Siegfried (1969), Dictionary of Science. New York: Dell.

McLaughlin, Peter (2001), What Functions Explain. Cambridge: Cambridge University Press.

Messer, William S., Jr. (2002), "Mbc 3320 Dopamine", http://www.neurosci.pharm.utoledo. edu/MBC3320/dopamine.htm.

Moran, Laurence A., K. Gray Scirmgeour, H. Robert Horton, Raymond S. Ochs, and J. David Rawn (1994), Biochemistry. Englewood Cliffs, NJ: Prentice Hall.

NCI (National Cancer Institute) (2002), "Making the Connections for a Targeted Cancer Treatment Takes Time and Perseverance", http://plan.cancer.gov/infra/sod.htm

Reznek, Lawrie (1987), The Nature of Disease. London: Routledge \& Kegan Paul.

Salmon, Wesley C. (1984), Scientific Explanation and the Causal Structure of the World. Princeton: Princeton University Press.

_ (1989), "Four Decades of Scientific Explanation", in Phillip Kitcher and Wesley C. Salmon (eds.), Scientific Explanation, Minnesota Studies in the History of Science, vol. 13. Minneapolis: University of Minnesota Press, 3-219.

Sapp, Shawn A. (2002), "Metamol Gallery", http://lamar.colostate.edu/ sasapp/metamolgallery.html.

Schaffner, Kenneth F. (1993), Discovery and Explanation in Biology and Medicine. Chicago: University of Chicago Press.

Schank, Roger C. (1986), Explanation Patterns: Understanding Mechanically and Creatively. Hillsdale, NJ: Erlbaum.

Steffan, Joan S. et al. (2001), "Histone Deacetylase Hinhiborts Arrest PolyglutamineDependent Neurodegeneration in Drosophila ital", Nature 413: 739-743.

Thagard, Paul (1988), Computational Philosophy of Science. Cambridge, MA: MIT Press/Bradford Books.

(1992), Conceptual Revolutions. Princeton: Princeton University Press. (1999), How Scientists Explain Disease. Princeton: Princeton University Press. (2002a), "Curing Cancer? Patrick Lee's Path to the Reovirus Treatment", International Studies in the Philosophy of Science 16: 179-193. (2002b), "How Molecules Matter to Mental Computation", Philosophy of Science 69: 429-446.

Therapeutics Initiative (2002), “Angiotensin II Receptor Blockers”, http://www.ti.ubc.ca/ pages/letter28.htm.

Woolfolk, Robert L. (1999), "Malfunction and Mental Illness”, Monist 82: 658-670. 